Journal of Engineering and Applied Sciences 14 (Special Issue 7): 10143-10151, 2019

ISSN: 1816-949X

(C) Medwell Journals, 2019

\title{
Study of the Effect of PANI Polymer on the Optical Properties of Malachite Green Dye
}

\author{
${ }^{1}$ Lazem H. Aboud, ${ }^{2}$ Rajaa K. Mohammad and ${ }^{2}$ Alaa H. Jassim \\ ${ }^{1}$ Department of Laser Physics, Science Collage for Women, University of Babylon, Hillah, Iraq \\ ${ }^{2}$ Department of Physics, College of Science, University of Kerbala, Karbala, Iraq
}

\begin{abstract}
In this research involves the study the optical properties of malachite green dye films prepared by laser pulse deposition method including absorbance, transmittance, absorption coefficient, refractive index and energy gap. This research also involves the study of the optical properties of malachite green dye films doping with PANI polymer and its effect on optical properties also involves some opinions and discussions that are related to the clarification of the effect of PANI polymer on the optical propertiesfor this dye.
\end{abstract}

Key words: Laser pulse deposition method, malachite-green dye, PANI polymer, liner optical properties, laser dyes, properties

\section{INTRODUCTION}

Malachite green (it is also called aniline green, Benz aldehyde green) has the chemical formula $\left(\mathrm{C}_{23} \mathrm{H}_{25} \mathrm{ClN}_{2}\right)$ and the molar mass (364.911 g/mol) as shown in Fig. 1, triphenylmethane dilute dye which is used medicinally in solution as a local antiseptic. Malachite green is used as an active laser medium, effective against fungi and bacteria. It was used to control the fungus saprolegnia that kills eggs and young fry in the field of fish breeding (Gessner and Mayer, 2000). Malachite green has frequently been used to catch thieves and pilferers. The bait, usually money is sprinkled with the anhydrous powder. When some put has hand on the contaminated money when he washshis hands he will notice a green spots on the skin that lasts for several days (Srivastava et al., 2004). Polymers are large molecules made up of small, interconnected units called the monomer. Polymers are sometimes crystalline, sometimes noncrystalline or a mixture of the two (Schultz, 1974).
Most polymers lack good electrical conductivity, so, their applications are limited to their chemical and mechanical properties. The electrical uses of polymers have been limited to electrical insulators because they have good electrical insulation properties (Moore, 1964).

It is possible to increase the electrical conductivity of some conductive polymers by increasing the evaporation rate. Among the polymers that are connected is the polyniline polymer (PANI polymer) which is considered an important polymer and it is characteristic of this polymer being soluble in a conductive form which is thermally and environmentally stable (Jayakannan et al., 2006). PANI polymer has high electrical properties (in the process of protonization) with proton acids. PANI polymer is either in the form of an insulation (base) or salt (conductive)(Murtagh and Starck, 1999).

In this study, we used one of these conductive polymers (PANI polymer) and study its effect on the optical properties of malachitegreen dye films.

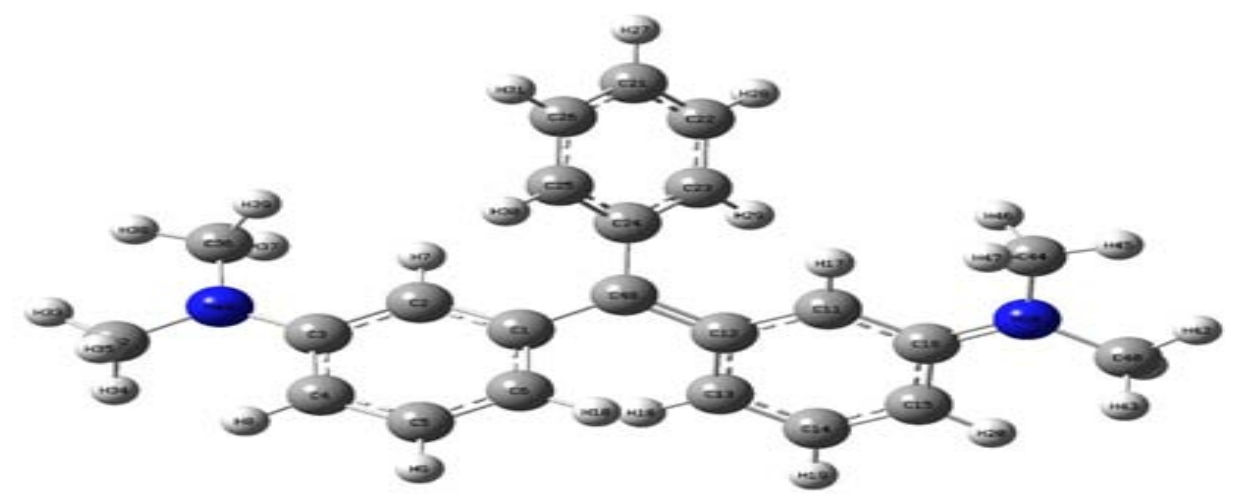

Fig. 1: Molecular structure of malachite green dye

Corresponding Author: Lazem H. Aboud, Department of Laser Physics, University of Babylon, Science Collage for Women, Hillah, Iraq 
There are many studies to study the optical properties of different dyes including in 2012, prepared a thin film of PolyMerase (PMMA) with different concentrations and measured the spectral properties of these films where the relative strength of absorption and fluorescence spectra was increased by increasing concentration (Al-Dulaimi, 2017). In 2013 study which studied the effect of dopping on the optical constants of (PMMA) dopping with phenolphthalein (8\%), the absorption, transmission, the reflectivity, extinction coefficient, refractive index and real and imaginary parts of dielectric constant were studied. The results were shown that all properties that were studied increases by the ratio of dopping except transmission which decreased at dopping (Azzawi, 2013). In 2017 study which studied the spectral properties of the rhodamine dye after the addition of polymer PVP (Abdullah, 2017). In 2018 study which studied the linear and nonlinear properties of membranes of polyvinyl alcohol inlaid with the dye of sodium fluorsin at different concentration (Tahir and Tawil, 2018). In 2018 prepared Polyvinyl Alcohol (PVA) with sodium fluorine and used as laser optical filters (Yahia et al., 2018). Figure 1 shows the molecular structure of the malachite green dye which used in this research, drawn by the (Gaussian 03) program.

\section{Liner optical properties}

Absorption (A): It is defined as the ratio between the intensity absorbed beam with absorbed by the matter to the original intensity incident beam on it and gives the ratioas follows (Callister, 2001; Tahir and Al-Yasari 2018):

$$
\mathrm{A}=\frac{\mathrm{I}_{\mathrm{A}}}{\mathrm{I}_{\mathrm{o}}}
$$

Reflection (R): It is defined as the bounce of light falling on a surface that separates two different energies in optical density as follows (Li et al., 2008):

$$
R=\left(\frac{n-1}{n+1}\right)^{2}
$$

The Reflectance value (R) can be calculated by using the following equation:

$$
\mathrm{R}=1-(\mathrm{A}+\mathrm{T})
$$

Where $\mathrm{A}$ is the absorbance.

Transmittance (T): It is defined as the percentage of light intensity applied to the intensity of the falling light. It is given as follows (Parikh, 1974, Demtroder, 2010):

$$
\mathrm{T}=\left(\frac{\mathrm{I}}{\mathrm{I}_{\mathrm{o}}}\right)
$$

According to the Per-Lambert act, transmittance decreases as the molar concentration increases and the length of the optical path L passes through the light (Straughan and Walker, 1976).

Absorption coefficient $\left(\boldsymbol{\alpha}_{\mathbf{0}}\right)$ : It is the ratio of decrease in the incident beam on the matter of accordance with the distance towards spread the wave inside the matter. Which can be found from the curved transmission (Sivanesan et al., 2010):

$$
\alpha_{0}=2.303\left(\frac{\mathrm{A}}{\mathrm{d}}\right)
$$

Where:

$\alpha_{0}$ : The absorption coefficient is calculated using the above equation at any given wavelength

$\mathrm{d}$ : The thickness and its value is $1 \mathrm{~cm}$

Refractive index: It is an important parameter for optical materials and applications. Thus, it is important to determine optical constants of the crystal. The refractive index of the crystal can be easily calculated from the reflectance values (Abdullah et al., 2013):

$$
\mathrm{n}=\left[\llbracket \frac{4 \mathrm{R}-\mathrm{K}^{2}}{(\mathrm{R}-1)^{2}}\right] \| 0.5-\frac{\mathrm{R}+1}{\mathrm{R}-1}
$$

Where:

$\mathrm{R}$ : The reflectance

$\mathrm{K}$ : The extinction coefficient

Extinction coefficient: It is the quantity of energy that absorbed by the electronics valance band at the incident of beam on the matter (Sirotin and Shasko-Skaia, 1982).

\section{MATERIALS AND METHODS}

The malachite green dye was used due to its importance in many applications, especially because it functions as an active medium in dye lasers. Table1 shows some physical properties of dye as well as Table 2 showing the physical properties of PANI polymer.

\section{Experimental part}

Preparation of samples

Capping samples: PNVI polymer powder has been compressed into pellets using the Chinese hydraulic piston with a (10 ton) strength shown in Fig. 2. The piston 


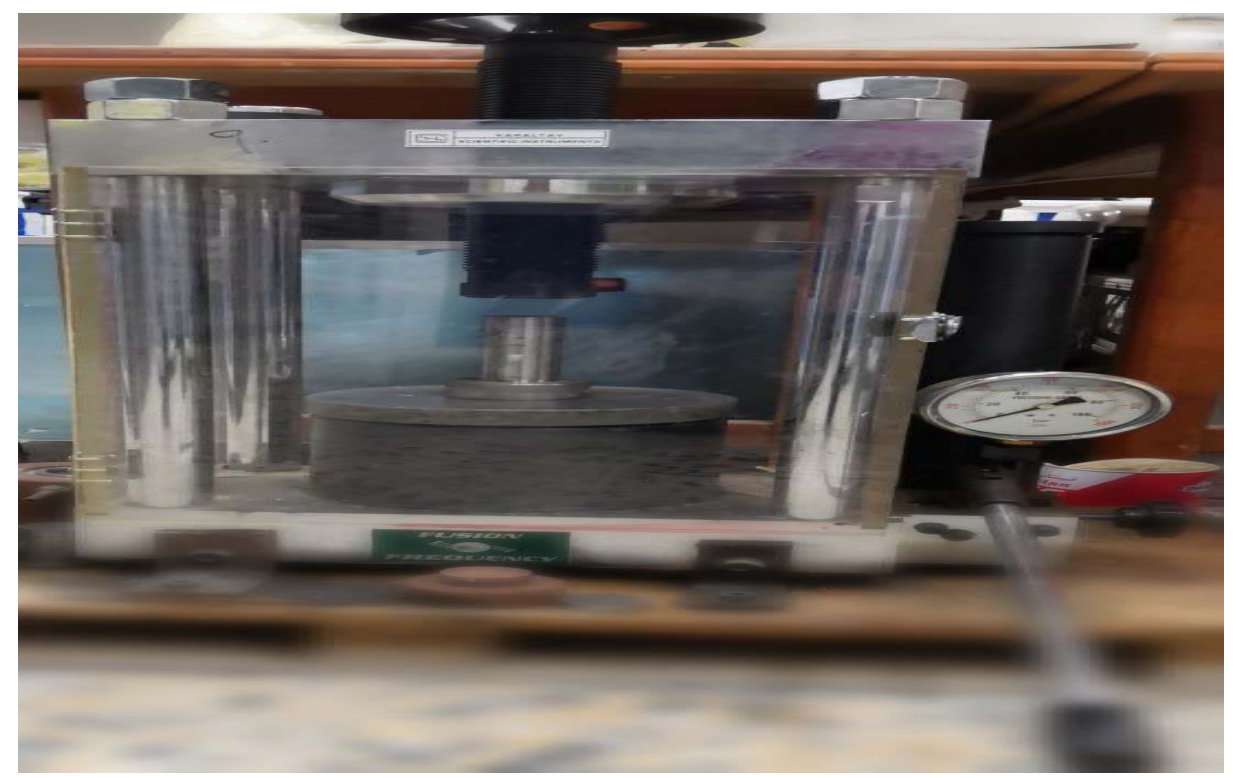

Fig. 2: Hydraulic piston

Table 1: Physical properties of the malachite green dye (O'Neil, 2013; USEPA., 2010)

\begin{tabular}{|c|c|}
\hline Dye & Malachite green \\
\hline The scientific name & $\begin{array}{l}\text { Aniline green, benzaldehyde green } \\
\text { or china green }\end{array}$ \\
\hline Chemical formula & $\mathrm{C}_{23} \mathrm{H}_{25} \mathrm{ClN}_{2}$ \\
\hline $\begin{array}{l}\text { Molecular weight } \\
\text { (g/mol) }\end{array}$ & 364.911 \\
\hline Uses & $\begin{array}{l}\text { Laser active media, dyeing silk, wool, } \\
\text { biological staining, etc. }\end{array}$ \\
\hline Color & Green crystals with metallic luster \\
\hline Solubility & $\begin{array}{l}\text { Sol in alcohol, methanol, chloroform, } \\
\text { amyl alcohol }\end{array}$ \\
\hline & In water, $4.0 \times 10^{4} \mathrm{mg} / \mathrm{L}$ at $25^{\circ} \mathrm{C}$ \\
\hline Vapor pressure & $2.4 \times 10^{-13} \mathrm{~mm} \mathrm{Hg}$ at $25^{\circ} \mathrm{C}$ \\
\hline
\end{tabular}

Table 2: Physical properties of PANI polymer (MacDiarmid et al., 1987)

\begin{tabular}{ll}
\hline Polymer & Poly aniline \\
\hline Chemical formula & $(\mathrm{C} 6 \mathrm{H} 4 \mathrm{NH}) \mathrm{n}$ \\
Electrical conductivity & $30-200 \mathrm{~S} / \mathrm{cm}$ \\
Energy gap (eV) & 3.2 \\
Solvent & Distilled water \\
Color & Solid crystals of black color \\
\hline
\end{tabular}

consists of a small steel sheet used to put the material to be compressed. In addition, it is surrounded by a thick glass barrier to protect the user from the possibility of cracking the mold. The piston contains hydraulic oil that increases the pressure which can be read while moving the pressure lever using the meter scale. Steel molds may be used in different sizes depending on the size of the sample. The disk thickness of the polymer was about $5 \mathrm{~mm}$ and $4 \mathrm{~cm}$ diameter, allowing the possibility of using more sites than the hard disk surfaces in the deposition processes to obtain more homogeneous films.

Deposition of PANI polymer films in pulse laser deposition method: The pulsed laser deposition method was used to prepare thin films for nanoparticle particles.
This system consists of the main parts shown in Fig. 3, consisting of the discharge chamber, the Nd-YAG pulse laser with a wavelength of $1064 \mathrm{~nm}$, a convex lens made of quartz by dimension $30 \mathrm{~cm}$ focal length, quartz glass window, pressure control screen, rotary pump, spread pump, there are a set of steps we follow to prepare membranes which are as follows:

Clean the vacuum chamber using acetone alcohol and a soft cloth with attention to leaving the room for $24 \mathrm{~h}$ exposed to air place the 4 glass flooring on the slide holder, so that, it is against the target on the rotor target holder, so that, the distance between the target and the ground is $4 \mathrm{~cm}$

The Nd-YAG laser beam focuses on $100 \mathrm{mj}$ for dye and $80 \mathrm{~mJ}$ for dye doped with PANI polymer 25, 50, 75 pulse for the dye and 100, 200, 300 pulse for dye doped with PANI polymer using a convex lens with a focal distance of $30 \mathrm{~cm}$ on the rotor target at a $45^{\circ}$ angle.

Close the discharge chamber with all the valves closed and start the discharge by opening the rotary pump valve and when the pressure reaches $10-^{3}$ mbar. The rotary pump valve is closed and open the pump valve spread until the room vacuum reaches $10-{ }^{5}$ mbar.

Heat the floor using a halogen lamp until the temperature reaches $250^{\circ} \mathrm{C}$ to increase the adhesion of the material to the ground. After the desired discharge is achieved while the vacuum chamber valve remains open, the laser pulses are given. The result is the plasma state that contains the particles that will be deposited on the floor.

The films are obtained with a malachite green dye or with dye doping PANI polymer material on the glass floors and undergo optical properties, structural and electrical tests. 


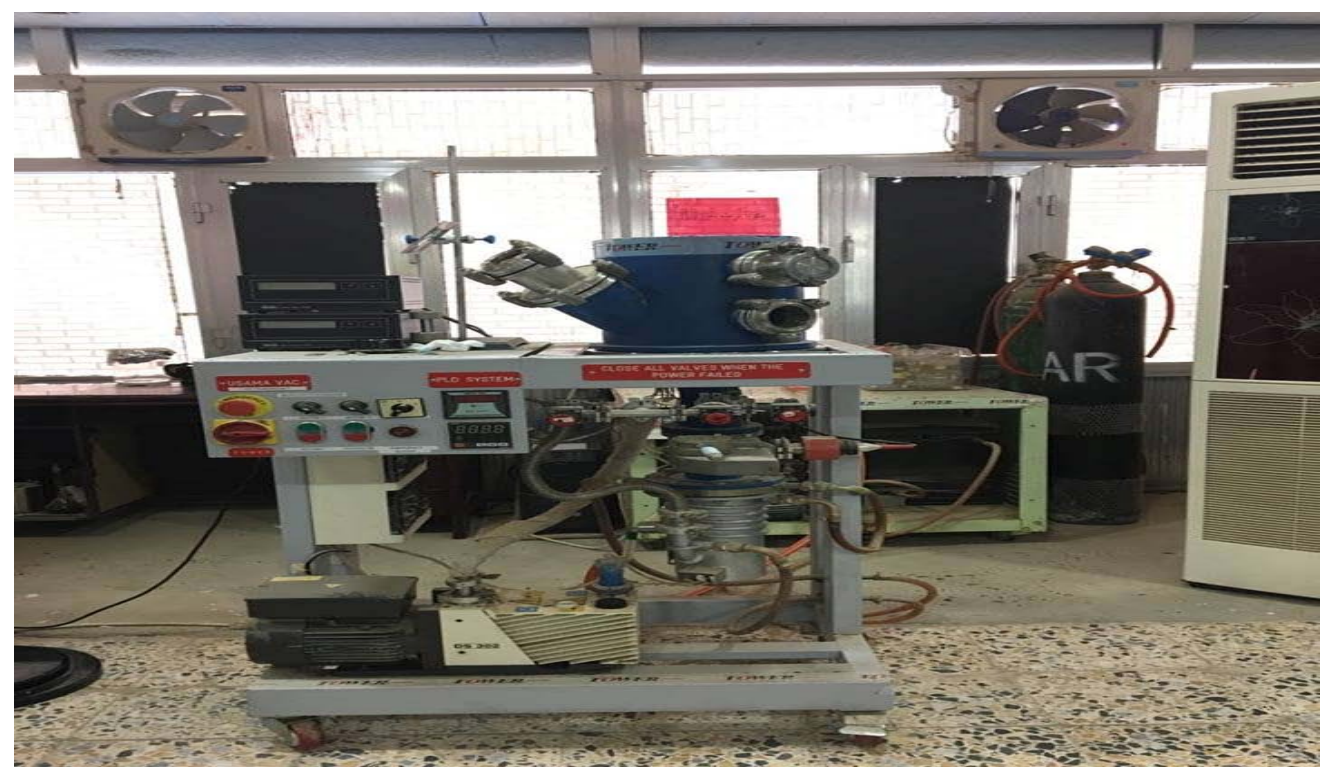

Fig. 3: Pulse laser deposition system

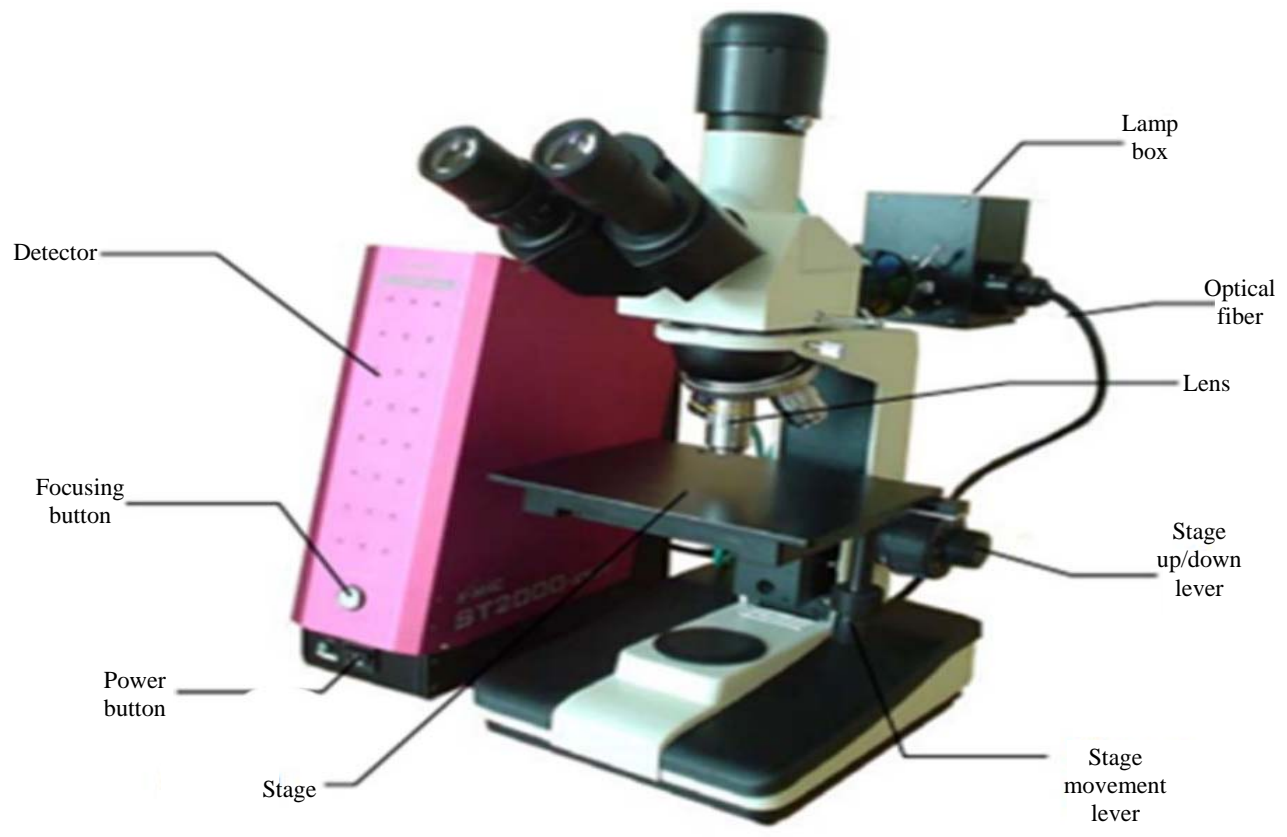

Fig. 4: Measuring devices for thin-film thickness

Thickness measurement: There are several ways to measure the thickness of thin films based on mechanical, optical or microscopic methods. In this study, the device is shown in Fig. 4. This device depends on the optical path. Therefore, the interference phenomenon between light reflected from the film and light reflected from the base or the phase difference between the reflected radii is what determines the properties of the thin film. In this way, we can not only calculate the thickness of the film as well as the optical constants of the film. If the film is transparent and there is visual interference, the thickness can be calculated for any sample as well if the multilayer film can calculate the thickness of each layer Fig. 5.

Principle of work: The light beam emits a tungsten-halogen lamp and goes through the optics of the microscope and then falls on the film. The reflected light from the surface separates the film from the base and enters series spectra tkick through the optical fiber, located at the center of the probe which collects the 


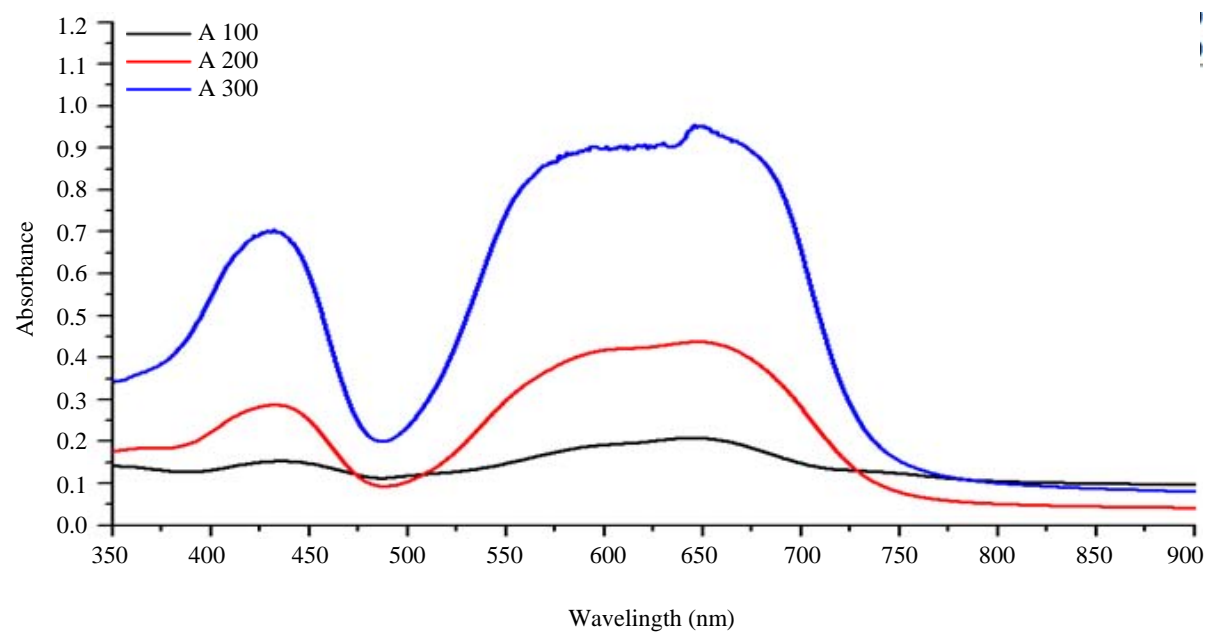

Fig. 5: Absorption spectra of pure malachite green dye films

reflected light. This reflected light is amplified by this chain and converted to an electrical signal by the CCD camera that the wavelengths are then converted to a digital signal by the $\mathrm{A} / \mathrm{D}$ adapter and transferred to the computer. The probe reflection sensor contains 6 additional fibers around its perimeter that help locate the light and find the exact location on the sample to be measured.

Measured signals may contain noise resulting from several factors, so, inaccurate measurement results are obtained. In order to reduce the noise effect on the signal, the series spectra thick eliminates noise by calculating the averages for both time bands and wavelengths. More reliable results for signal-free noise. The part of the light falling on the sample will be reflected from the top of the film while the other part reflected from the surface separating the film and base but in the case of the fact that the multi-layer film, the light falling will be reflected from the surfaces separating the different layers of the film and that this reflected radiation is similar. They are emitted from a single source, so that, the interference between them is either constructive or destructive depending on the wavelength. These reflective and interrelated rays give the shape of the spectrum represented by a sine wave or other, depending on the thickness of the sample and other optical constants. Through this spectroscopy the optical properties of the film can be obtained such as thick, refractive index, absorpation coefficient and others.

\section{RESULTS AND DISCUSSION}

Absorption spectra for pure malachite green dye films: The absorption spectra of malachite green dye films, its recorded by way of laser pulse deposition were studied using the (UV-Vis.) spectroscopy as shown in Fig. 5 and Table 3. From the absorbance spectra in Fig. 5, it was observed that the intensity of absorption is increases by increasing the number of laser pulses. The result of absorption spectra, transmittance spectra can be obtained as shown in Fig. 6 and Table 4.

It was observed in Fig. 6 the intensity of transmittance is decreases by increasing the number of laser pulses. After obtaining the transmittance results, absorption coefficient $\left(\alpha_{0}\right)$ and refractive index $\left(n_{0}\right)$ of the prepared samples after the introduction of the Eq. 5, 6. It is observed that the increasing in number of laser pulses leads to a increase in refractive index and absorption coefficient as shown in Table 5 and 6.

The energy gap for malachite green dye films was calculated for the highest wavelength and for the three pulses as in Fig. 7 and Table 7. Absorption spectra for films of malachite green dye doping with PANI polymer. The absorption spectra for films of malachite green dye doping with PANI polymer, its recorded by way of laser pulse deposition were studied using the (UV-V is.) spectroscopy as shown in Fig. 8 and Table 8. From the absorbance spectra in Fig. 8, it was observed that the intensity of absorption is increases by increasing the number of laser pulses. The result of absorption spectra, transmittance spectra can be obtained as shown in Fig. 9 and Table 9.

It was observed in Fig. 9 the intensity of transmittance is decreases by increasing the number of laser pulses. after obtaining the transmittance results, absorption coefficient $\left(\alpha_{0}\right)$ sand refractive index $\left(n_{0}\right)$ of the prepared samples after the introduction of Eq. 5 and 6. It is sobserved that the increasing in number of laser pulses leads to a decrease in refractive index and increase in absorption coefficient as shown in Table 10 and 11.

The energy gap for malachite green dye doping with PANI polymer films was calculated for the highest wavelength and for the three pulses as in Fig. 10 and Table 12. 


\section{J. Eng. Applied Sci., 14 (Special Issue 7): 10143-10151, 2019}

Table 3: Absorbance of only malachite green dye films

\begin{tabular}{|c|c|c|c|c|c|}
\hline \multicolumn{2}{|l|}{$100 \mathrm{P}$} & \multicolumn{2}{|l|}{$200 \mathrm{P}$} & \multicolumn{2}{|l|}{$300 \mathrm{P}$} \\
\hline$\underline{\lambda \max (\mathrm{nm})}$ & $\mathrm{A}$ & $\lambda \max (\mathrm{nm})$ & $\mathrm{A}$ & $\lambda \max (\mathrm{nm})$ & $\mathrm{A}$ \\
\hline 435 & 0.15325 & 433 & 0.2875 & 432 & 0.7055 \\
\hline$\underline{645}$ & 0.20875 & 647 & 0.43825 & 646 & 0.9540 \\
\hline \multicolumn{2}{|l|}{$100 \mathrm{P}$} & \multicolumn{2}{|l|}{$200 \mathrm{P}$} & \multicolumn{2}{|l|}{$300 \mathrm{P}$} \\
\hline$\lambda \max (\mathrm{nm})$ & t-values & $\lambda \max (\mathrm{nm})$ & $\mathrm{t}$-values & $\lambda \max (\mathrm{nm})$ & $\mathrm{t}$-values \\
\hline 435 & 0.702668 & 433 & 0.515822 & 432 & 0.197015 \\
\hline 645 & 0.618372 & 647 & 0.364544 & 617 & 0.111173 \\
\hline \multicolumn{2}{|l|}{$100 \mathrm{P}$} & \multicolumn{2}{|l|}{$200 \mathrm{P}$} & \multicolumn{2}{|l|}{$300 \mathrm{P}$} \\
\hline$\lambda \max (\mathrm{nm})$ & $\alpha\left(\mathrm{cm}^{-1}\right)$ & $\lambda \max (\mathrm{nm})$ & $\alpha\left(\mathrm{cm}^{-1}\right)$ & $\lambda \max (\mathrm{nm})$ & $\alpha\left(\mathrm{cm}^{-1}\right)$ \\
\hline 435 & 0.0003715 & 433 & 0.662113 & 432 & 0.0102963 \\
\hline$\underline{645}$ & 0.0050605 & 647 & 0.007476 & 646 & 0.0139230 \\
\hline \multicolumn{2}{|l|}{$\frac{1 \mathrm{duIe} 0}{1 \times 10^{-4}}$} & \multicolumn{2}{|l|}{$0.5 \times 10-4$} & \multicolumn{2}{|l|}{$0.2 \times 10^{-4}$} \\
\hline$\lambda \max (\mathrm{nm})$ & $\mathrm{n}$ & $\lambda \max (\mathrm{nm})$ & $\mathrm{n}$ & $\lambda \max (\mathrm{nm})$ & $\mathrm{n}$ \\
\hline$\overline{435}$ & 1.739115 & 433 & 2.127462 & 432 & 2.898955 \\
\hline 645 & 1.978772 & 647 & 2.286227 & 646 & 2.967996 \\
\hline
\end{tabular}

Table 7: The energy gap of pure malachite green dye films

No. of pulses (p)

$\mathrm{EG}(\mathrm{ev})$

100

200

2.3

300

Table 8: Transmittance for films of malachite green dye doping with PANI polymer

\section{$100 \mathrm{P}$}

$\underline{\max (\mathrm{nm})}$

421

640

Table 9: Transmittance for films of malachite green dye doping with PANI polyme

\begin{tabular}{|c|c|c|c|c|c|}
\hline \multicolumn{2}{|l|}{$100 \mathrm{P}$} & \multicolumn{2}{|l|}{$200 \mathrm{P}$} & \multicolumn{2}{|l|}{$300 \mathrm{P}$} \\
\hline$\lambda \max (\mathrm{nm})$ & t-values & $\lambda \max (\mathrm{nm})$ & t-values & $\lambda \max (\mathrm{nm})$ & t-values \\
\hline$\overline{421}$ & 0.271019 & 426 & 0.165196 & 424 & 0.107833 \\
\hline 640 & 0.246604 & 634 & 0.119193 & 628 & 0.105196 \\
\hline
\end{tabular}

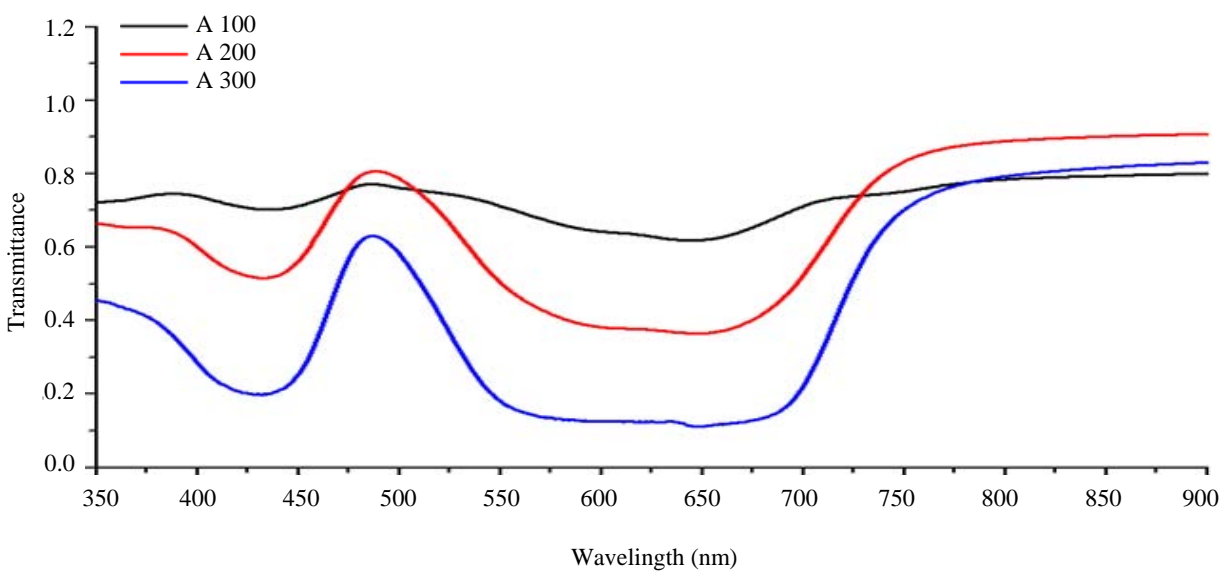

Fig. 6: Transmittance spectra of pure malachite green dye films 


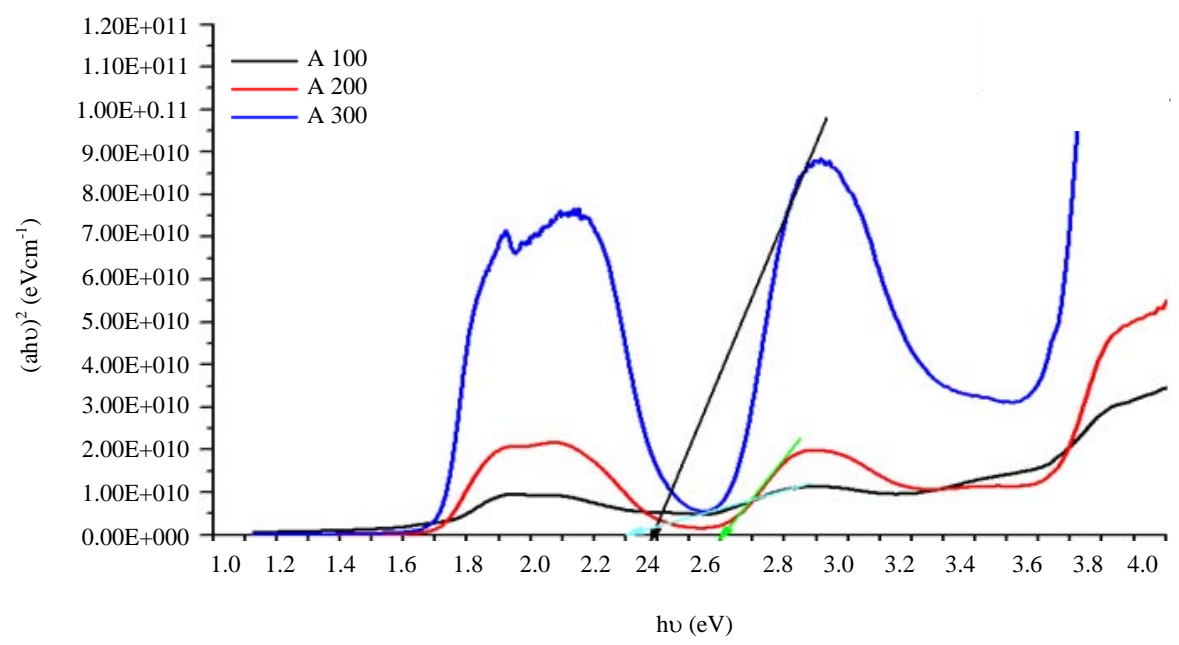

Fig. 7: The energy gap of pure malachite green dye films

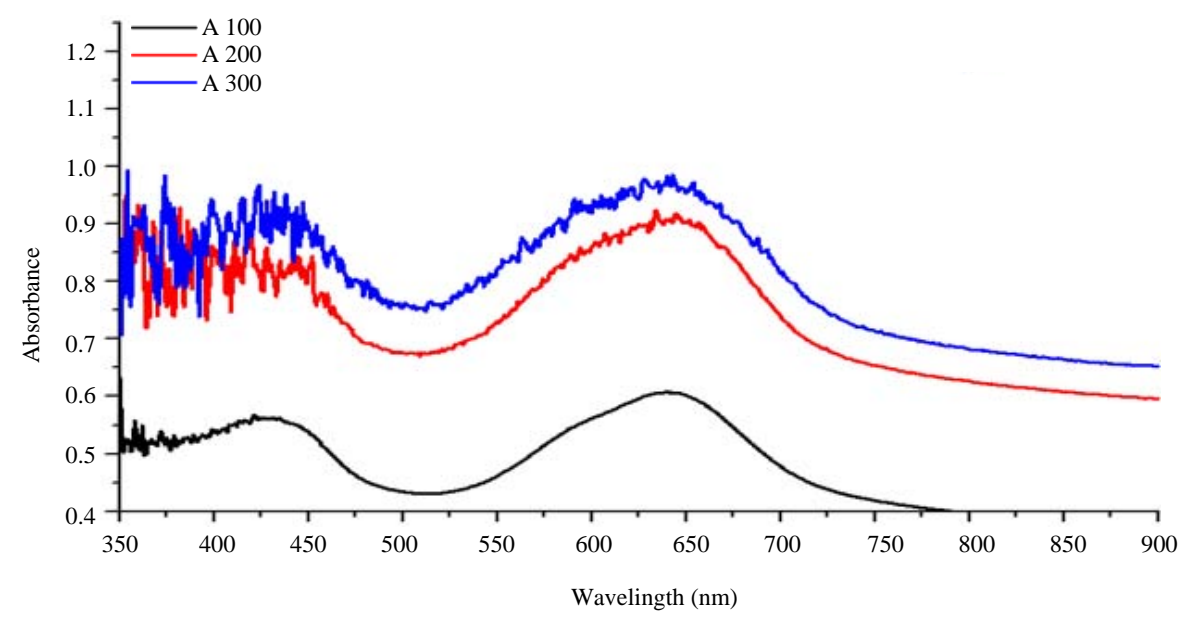

Fig. 8: Absorption spectra for films of malachite green dye doping with PANI polymar

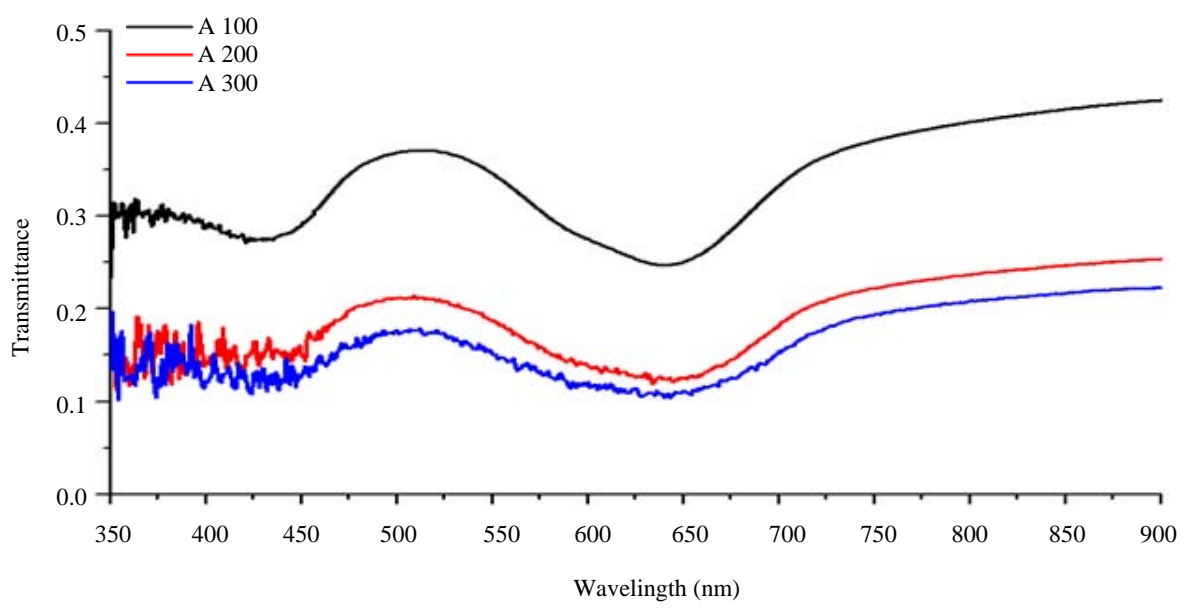

Fig. 9: Transmittance spectra for films of malachite green dye doping with PANI polymar 


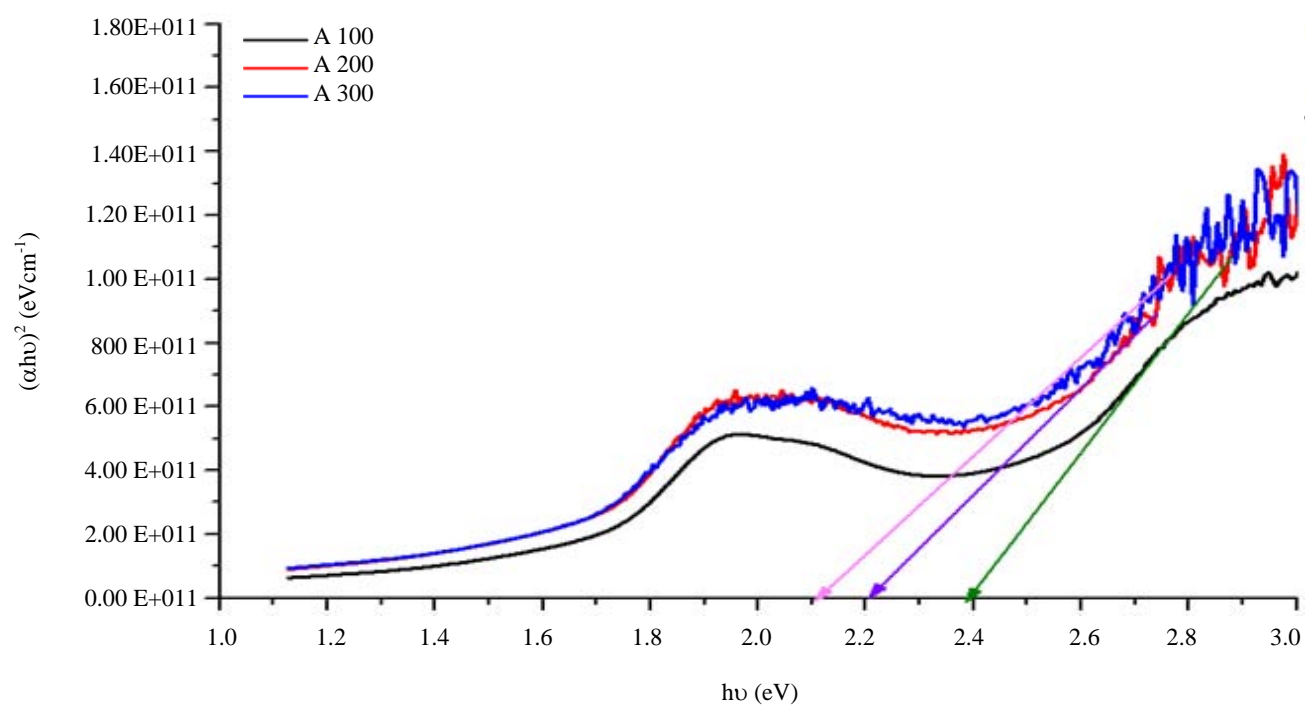

Fig. 10: The energy gap of malachite green dye doping with PANI films

Table 10: Absorption coefficient for films of malachite green dye doping with PANI polymer

\begin{tabular}{|c|c|c|c|c|c|}
\hline \multicolumn{2}{|l|}{$100 \mathrm{P}$} & \multicolumn{2}{|l|}{$200 \mathrm{P}$} & \multicolumn{2}{|l|}{$300 \mathrm{P}$} \\
\hline$\lambda \max (\mathrm{nm})$ & $\alpha\left(\mathrm{cm}^{-1}\right)$ & $\lambda \max (\mathrm{nm})$ & $\alpha\left(\mathrm{cm}^{-1}\right)$ & $\lambda \max (\mathrm{nm})$ & $\alpha\left(\mathrm{cm}^{-1}\right)$ \\
\hline$\overline{421}$ & 0.010836 & 426 & 0.011035 & 424 & 0.0125285 \\
\hline 640 & 0.0116201 & 634 & 0.0120355 & 628 & 0.0136678 \\
\hline
\end{tabular}

Table 11: Refractive index for films of malachite green dye doping with PANI polymer

\begin{tabular}{|c|c|c|c|c|c|}
\hline \multicolumn{2}{|l|}{$100 \mathrm{P}$} & \multicolumn{2}{|l|}{$200 \mathrm{P}$} & \multicolumn{2}{|l|}{$300 \mathrm{P}$} \\
\hline$\lambda \max (\mathrm{nm})$ & $\mathrm{n}$ & $\lambda \max (\mathrm{nm})$ & $\mathrm{n}$ & $\lambda \max (\mathrm{nm})$ & $\mathrm{n}$ \\
\hline 421 & 1.954596 & 426 & 1.250986 & 424 & 0.70661 \\
\hline 640 & 2.105999 & 634 & 0.786480 & 628 & 0.63089 \\
\hline
\end{tabular}

Table 12: The energy gap of malachite green dye doping with PANI polymer films

\begin{tabular}{lc}
\hline No. of pulses $(\mathrm{p})$ & $\mathrm{EG}(\mathrm{eV})$ \\
\hline 100 & 2.4 \\
200 & 2.2 \\
300 & 2.1 \\
\hline
\end{tabular}

\section{CONCLUSION}

The absorption of film increases with increasing of laser pulse, due to the increased thickness of the films. The values of absorption coefficient and refractive index of pure malachite green dye films increase with increasing number of laser pulses while the values of refraction coefficient for films of malachite green dye doping with PANI polymer decrease with increasing the number of laser pulses because of the polymer effect on the dye.

Two absorption peaks in the all dye films (two energy transitions) at the wavelengths $(\approx 424$ and $\approx 635 \mathrm{~nm}$ ) with large broadening at both peaks.

The broadening of absorption peaks results from the presence of many vibration and rotational energy levels of electronic energy levels and because of the effect of conductive polymer also on electronic energy levels. Energy gap of pure malachite green dye films range from 2.3-206 eV while energy gap of malachite green dye doping with PANI polymer declining to range 2.1-2.4 eV because of the effect of conductive polymer.

The appearance of deformation in purity of the absorbance spectra for films of malachite green dye doping with PANI polymer, especially, at the top of the $(\approx 424 \mathrm{~nm})$ with a slight deformation of about 600 . Because the curved at peak of $635 \mathrm{~nm}$ resisted deformation more stable. The deformation is increased by increasing the number of pulses, i.e., by increasing the polymer. This can beat tribute to the electrical properties of the PANI polymer and their effect on the electronic energy levels of the molecule.

\section{REFERENCES}

Abdullah, B.A., 2017. Optical properties of Rhodamine B dye mixed with Polyvinylpyrrolidone (PVP) as a matrix. J. Kufa Phys., 9: 11-19. 
Abdullah, O.G., B.K. Aziz and D.M. Salh, 2013. Structural and optical properties of PVA: $\mathrm{Na}_{2} \mathrm{~S}_{2} \mathrm{O}_{3}$ polymer electrolytes films. Indian J. Applied Res., 3: 477-480.

Al-Dulaimi, G.S.A., 2017. Preparing the thin films from laser dye dopped with polymer and measuring thickness. Ibn AL Haitham J. Pure Appl. Sci., 25: 220-228.

Azzawi, A.H.R., 2013. Dopping effect on optical constant of polymethylacrelate (PMMA) dopping with phenolphthalein. J. Univ. Babylon, 21: 1457-1465.

Callister, W.D., 2005. Fundamentals of Materials Science and Engineering: An Integrated Approach. 2nd Edn.,/Vol. 1, John Wiley \& Sons, Hoboken, New Jersey, USA., ISBN:9780471470144, Pages: 824.

Demtroder, W., 2010. Atoms, Molecules and Photons an Introduction to Atomic Molecular and Quantum Physics. 2nd Edn., Springer, Berlin, Germany, ISBN:978-3-642-10297-4, Pages: 589.

Gessner, T. and U. Mayer, 2000. Triarylmethane and diarylmethane dyes. Ullmanns Encycl. Ind. Chem., Vol. 1, 10.1002/14356007.a27_179

Jayakannan, M., P. Anilkumar and A. Sanju, 2006. Synthesis and characterization of new azobenzenesulfonic acids doped conducting polyaniline. Eur. Polym. J., 42: 2623-2631.

Li, J., M. Wang, Z. Lin, X. Huo and G. Zhai, 2008. Optical linearity and nonlinearity of ZnSe nanocrystals embedded in epoxy resin matrix investigated by Z-scan technique. Ceram. Intl., 34: 1073-1076.

MacDiarmid, A.G., J.C. Chiang, A.F. Richter and A.A. Epstein, 1987. Polyaniline: A new concept in conducting polymers. Synth. Met., 18: 285-290.

Moore, W.R., 1964. An introduction to polymer chemistry. Soil Sci., 97: 291-291.

Murtagh, F. and J.L. Starck, 1999. Image and object analysis based on wavelet and multiscale transforms and noise modeling: The MR/1 software package. Bull. Am. Astron. Soc., 31: 1245-1245.
O’Neil, M.J., 2013. The Merck Index: An Encyclopedia of Chemicals, Drugs and Biologicals. Royal Society of Chemistry, London, UK., ISBN:9781849736701,.

Parikh, V.M., 1974. Absorption Spectroscopy of Organic Molecules. Addison-Wesley, Boston, Massachusetts, USA., Pages: 325.

Schultz, J., 1974. Polymer Materials Science. Prentice Hall, Upper Saddle River, New Jersey, USA., ISBN:9780136870388, Pages: 524.

Sirotin, I.U.I. and M.P. Shasko-Skaia, 1982. Fundamentals of Crystal Physics. MIR Publishers, Moscow, Russia, Pages: 654.

Sivanesan, T., V. Natarajan and S. Pandi, 2010. Nonlinear optical properties of $\alpha$-glycine single crystals by Z-Scan technique. Indian J. Sci. Technol., 3: 653-655.

Srivastava, S., R. Sinha and D. Roya, 2004. Toxicological effects of malachite green. Aquat. Toxicol., 66: 319-329.

Straughan, B.P. and S. Walker, 1976. Molecular Quantum Numbers of Diatomic Molecules. In: Spectroscopy: Volume Three, Straughan, B.P. and S. Walker (Eds.). Springer, Dordrecht, ISBN:978-0-412-13390-9, pp: $1-25$.

Tahir, K.J. and F.A. Tawil, 2018. Study the optical properties of fluorescein Sodium dye doped in polymer poly polyvinyl alcohol for different thickness. J. Kerbala Univ., 16: 4-19.

Tahir, K.J. and R. Al-Yasari, 2018. Structural and optical properties of Polyvinyl Alcohol doped Silver (PVA: Ag) film. J. Nanostruct., 8: 359-365.

USEPA., 2010. Estimation Program Interface (EPI) suite. United States Environmental Protection Agency, Washington, D.C., USA. https://appdb.winehq.org/objectManager.php?sClas s=application\&iId $=14007$

Yahia, I.S., A. Bouzidi, H.Y. Zahran, W. Jilani and S. AlFaify et al., 2018. Design of smart optical sensor using polyvinyl alcohol/Fluorescein Sodium salt: Laser filters and optical limiting effect. J. Mol. Struct., 1156: 492-500. 